Rev Inv Vet Perú 2014; 25(2): 226-232

doi: http://doi.org/10.15381/rivep.v25i2.8495

\title{
PATRÓN ECOGRÁFICO DE LOS ÓRGANOS ABDOMINALES DEL CONEJO DOMÉSTICO (Oryctolagus cuniculus)
}

\author{
Echographic Pattern of the Abdominal Organs of the Domestic Rabbit \\ (ORYCTOLAGUS CUNICULUS)
}

\author{
Jacqueline Cahua U. ${ }^{1,4}$, Miryam Quevedo U. ${ }^{2}$, Jesús Lescano G. ${ }^{2}$, \\ José Bustamante L. ${ }^{3}$, Graciela Poma B. ${ }^{1}$
}

\section{Resumen}

El presente estudio tuvo por objetivo la determinación del patrón ecográfico cuantitativo de los órganos abdominales del conejo doméstico. Se trabajó con 16 conejos adultos (11 hembras y 5 machos) de raza Nueva Zelanda, obteniéndose las mediciones descriptivas de hígado (diafragma hilio, dorsoventral y diámetro transverso), vesícula biliar (longitud y espesor), pared gástrica, vejiga (pared, longitud y espesor), bazo (longitud, espesor y diámetro transverso), riñones (longitud, espesor y corteza), próstata (longitud, espesor y diámetro) y cuerpo uterino.

Palabras clave: lagomorfo, ultrasonografía, abdomen, conejo

\section{Abstract}

The present study aimed to determine the quantitative echographic pattern of abdominal organs of the domestic rabbit. Ultrasound examination was performed in $16 \mathrm{New}$ Zealand mature rabbits ( 11 females and 5 males). Descriptive measurements were obtained for liver (diaphragm hilum, dorsal ventral and transverse diameter), gallbladder (length and thickness), gastric wall, urinary bladder (wall, length and thickness), spleen (length,

${ }^{1}$ Clínica de Animales Menores, ${ }^{2}$ Laboratorio de Anatomía y Fauna Silvestre, ${ }^{3}$ Laboratorio de Producción Avícola y Especies Menores, Facultad de Medicina Veterinaria, Universidad Nacional Mayor de San Marcos, Lima

${ }^{4}$ E-mail:jackiecahua@gmail.com

Recibido: 3 de julio de 2013

Aceptado para publicación: 25 de noviembre de 2013 
thickness and transverse diameter), kidneys (length, thickness and cortex), prostate (length, thickness and diameter) and uterine body.

Key words: lagomorph, ultrasonography, abdomen, rabbit

\section{INTRODUCCIÓN}

Los conejos se han popularizado como animales de compañía y, por lo tanto, se presentan con frecuencia a la clínica veterinaria para consulta rutinaria y emergencias (Graham y Mader, 2012). Se les encuentra mayormente en áreas urbanas donde el espacio es limitado y existen restricciones para mantener perros y gatos (Stein y Walshaw, 1996; Van der Hage, 2000). El conejo se ha convertido en la tercera mascota más común (después del perro y el gato), posiblemente debido a factores conductuales y de manejo (Richardson, 2000), de allí que la demanda de servicios para el conejo en la clínica veterinaria, incluyendo el diagnóstico por imágenes, se haya incrementado. Asimismo, los avances tecnológicos han permitido la mejora de la calidad de estos servicios (Reese, 2010).

Los conejos pueden padecer de enfermedades que afectan a los órganos de la cavidad abdominal, siendo más frecuentes las afecciones del aparato gastrointestinal (estasis gastrointestinal, enteritis), seguido por el aparato urinario (urolitos, cistitis), patologías hepáticas (hepatosis grasa, hepatitis) y neoplasias (Lammers y Winkelmann, 1997). Asimismo, las hembras pueden presentar problemas reproductivos, especialmente adenocarcinomas, piometras e hidrometras. Estas patologías puede ser diagnosticadas y diferenciadas mediante ultrasonografía (Redrobe, 2001).

La ultrasonografía es una técnica no invasiva y segura, utilizada frecuentemente en la práctica veterinaria, permitiendo al clí- nico obtener información instantánea sobre un amplio abanico de sistemas corporales. En determinados casos, la ecografía permite evaluar la función dinámica de algunos órganos, facilita en algunos casos la realización de biopsias y permite mejorar los conocimientos sobre anatomía y diversos procesos fisiológicos (Goddard, 2000; Meredith, 2000; Fischetti, 2012). Sin embargo, se requiere ampliar la información sobre el patrón normal y anormal de la anatomía a través del uso de la ultrasonografía en el conejo doméstico $(O$. cuniculus). El objetivo del presente estudio fue describir el patrón ultrasonográfico cuantitativo de los órganos abdominales del conejo doméstico.

\section{Materiales y MéTodos}

Se trabajó con 15 conejos adultos de raza Nueva Zelanda (11 hembras y 5 machos) de la granja de conejos de la Facultad de Medicina Veterinaria (FMV) de la Universidad Nacional Mayor de San Marcos, en Lima. El criterio de inclusión fue la ausencia de signos de enfermedad mediante examen clínico. Los animales recibían alimento comercial peletizado a base de alfalfa.

El examen ecográfico fue practicado en la Clínica de Animales Menores, FMVUNMSM. Se empleó un ecógrafo SonoAce Pico (Samsung Medison, Korea) con transductor microconvexo de 4-9 MHz. No fue necesaria la administración de drogas tranquilizantes a los animales experimentales. $\mathrm{La}$ evaluación de cada animal se hizo en cerca de 25 minutos y siempre fue ejecutada por el mismo evaluador. 
Cada conejo fue pesado durante los minutos previos al inicio de la evaluación ultrasonográfica, utilizando una balanza digital EBSA-20 (Zhongshan Jinli Electronic Weighing Equipment, China) con capacidad máxima de $20 \mathrm{~kg}$ y precisión de $5 \mathrm{~g}$. Asimismo, se estimó la condición corporal de los individuos empleando una escala del 1 al 5, la cual fue adaptada de la escala presentada por Cardinalli et al. (2008).

Para el examen ecográfico, los conejos fueron colocados en posición decúbito dorsal, sobre una colchoneta de soporte en « $\mathrm{V} »$ y con contención física del tórax, cadera y miembros (Reese, 2010). Se rasuró un área de $10 \times 6 \mathrm{~cm}$ en el abdomen, tomando como márgenes el borde caudal de las últimas costillas, el pliegue de los flancos y el pubis. Se aplicó gel de acoplamiento acústico y se procedió a la evaluación tomando como base el esquema de anatomía ultrasonográfica normal del conejo (Redrobe, 2006). Se determinó la situación, forma, tamaño y ecoestructura del parénquima de riñones, vejiga, útero, estómago, intestino y bazo aplicando cortes sagitales, transversales y oblícuos. Asimismo, se tomaron las siguientes medidas (en milímetros): hígado (diafragma hilio, dorso-ventral, diámetro transverso), vesícula biliar (longitud, espesor), estómago (espesor de mucosa), intestino delgado (espesor de pared), ciego (espesor de pared), vejiga (espesor de pared, longitud, espesor total), bazo (longitud, espesor, diámetro transverso), riñones (longitud, espesor, corteza), y próstata (longitud, espesor, diámetro transverso). Cada una de estas mediciones se hizo por triplicado durante el examen de cada individuo.

La medida diafragma - hilio hepático se tomó desde la cúpula del hígado hasta la zona hiliar, midiendo así el espesor del hígado. La medida dorsoventral hepática se tomó desde la cara ventral del hígado hasta la cara dorsal, teniendo a la aorta abdominal como límite, trazando una línea perpendicular a la aorta y que pase por la zona hiliar. Asimismo, para medir el dorsoventral en transverso he- pático se tomó el espesor del hígado de dorsal a ventral en un corte transverso del animal. La longitud de la vesícula biliar fue medida entre el inicio del conducto cístico y el extremo opuesto, mientras que el espesor fue medido entre las paredes laterales de este órgano.

Las medidas renales se tomaron a través de un corte sagital. La longitud se midió entre los extremos craneal y caudal y el espesor entre los bordes medial y lateral. La corteza se midió de lateral a medial tomando como referencia su hipoecogenicidad y teniendo como límite la anecogenicidad de la médula renal, mientras que la longitud vesical fue desde la abertura uretral hasta el polo craneal de la vejiga y el espesor vesical se midió de dorsal a ventral.

La longitud de la próstata se midió entre el polo craneal y el polo caudal, el espesor entre sus bordes ventral y dorsal, y el diámetro transverso entre sus bordes laterales. El cuerpo del útero se midió en un corte transverso que incluía las paredes uterinas dorsal y ventral. La longitud esplénica se midió entre los extremos lateral y medial, el diámetro transverso entre los bordes craneal y caudal y el espesor entre sus superficies dorsal y ventral.

Los datos de mediciones se procesaron mediante estadística descriptiva de tendencia central y dispersión.

\section{Resultados y Discusión}

El peso corporal de los conejos fue 3.5 $\pm 0.48 \mathrm{~kg}$ (rango de 2.7 a $4.3 \mathrm{~kg}$ ), con condición corporal de 3 en una escala de 1 a 5 . Los valores promedio de las medidas ecográficas de los órganos abdominales se presentan en el Cuadro 1 y las imágenes representativas se muestran en la Fig. 1. Cabe señalar que los animales presentaron la vejiga llena de líquido y el estómago con contenido alimenticio de superficie hiperecogénica, 
Cuadro 1. Medidas ecográficas $(\mathrm{mm})$ de órganos abdominales de conejos Nueva Zelanda (11 hembras y 5 machos)

\begin{tabular}{|c|c|c|c|c|}
\hline Órgano & $\begin{array}{l}\text { Observaciones } \\
\text { (n) }\end{array}$ & Media & $\begin{array}{l}\text { Desviación } \\
\text { estándar }\end{array}$ & Rango \\
\hline \multicolumn{5}{|l|}{ Hígado } \\
\hline Diafragma hilio & 15 & 37.6 & 8.4 & $22.9-52.5$ \\
\hline Dorsoventral & 15 & 30.5 & 4.3 & $22.8-39.7$ \\
\hline $\begin{array}{l}\text { Dorsoventral en } \\
\text { transverso }\end{array}$ & 11 & 30.8 & 4.0 & $24.2-36.8$ \\
\hline \multicolumn{5}{|l|}{ Vesícula biliar } \\
\hline Longitud & 8 & 24.8 & 7.5 & $18.0-38.0$ \\
\hline Espesor & 14 & 9.7 & 2.9 & $4.7-13.8$ \\
\hline \multicolumn{5}{|l|}{ Riñón izquierdo } \\
\hline Longitud & 16 & 39.6 & 2.1 & $37.0-44.2$ \\
\hline Espesor & 16 & 20.0 & 2.0 & $19.0-22.0$ \\
\hline Corteza & 16 & 3.4 & 0.4 & $3.0-4.1$ \\
\hline \multicolumn{5}{|l|}{ Riñón derecho } \\
\hline Longitud & 16 & 40.3 & 2.0 & $37.6-42.6$ \\
\hline Espesor & 16 & 18.8 & 1.1 & $17.1-20.4$ \\
\hline Corteza & 16 & 3.4 & 0.3 & $3.0-3.9$ \\
\hline \multicolumn{5}{|l|}{ Vejiga } \\
\hline Pared & 14 & 1.5 & 0.3 & $1.2-2.1$ \\
\hline Longitud & 14 & 55.3 & 22.2 & $32.2-82.4$ \\
\hline Espesor & 13 & 25.0 & 9.7 & $13.1-41.4$ \\
\hline \multicolumn{5}{|l|}{ Bazo } \\
\hline Longitud & 14 & 23.4 & 6.6 & $18.8-28.7$ \\
\hline Espesor & 15 & 5.1 & 0.6 & $4.6-6.5$ \\
\hline Diámetro transverso & 5 & 9.2 & 0.7 & $8.1-9.9$ \\
\hline \multicolumn{5}{|l|}{ Próstata } \\
\hline Longitud & 2 & 17.9 & 5.2 & $14.2-21.6$ \\
\hline Espesor & 2 & 10.0 & 1.4 & $9.0-11.0$ \\
\hline Diámetro transverso & & 13.8 & 1.3 & $12.9-14.6$ \\
\hline Cuerpo uterino & 4 & 4.3 & 0.8 & $3.2-4.9$ \\
\hline Pared estomacal & 16 & 1.9 & 0.4 & $1.4-2.7$ \\
\hline Pared intestinal & 14 & 1.8 & 03 & $1.3-2.1$ \\
\hline Pared cecal & 13 & 1.6 & 0.3 & $1.1-2.0$ \\
\hline
\end{tabular}




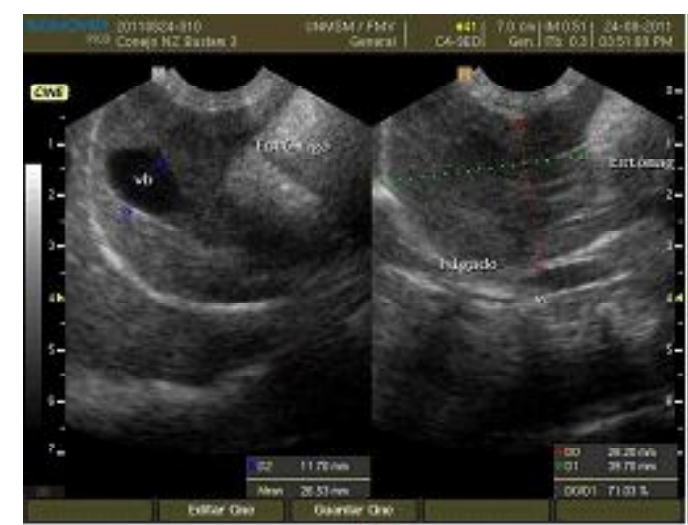

(A)

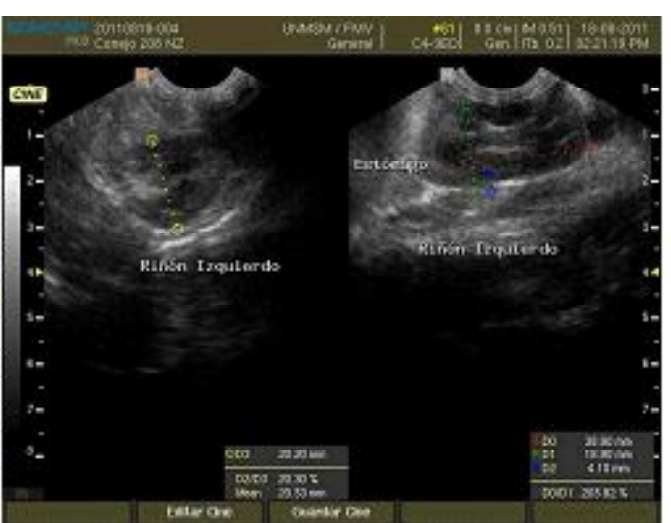

(B)

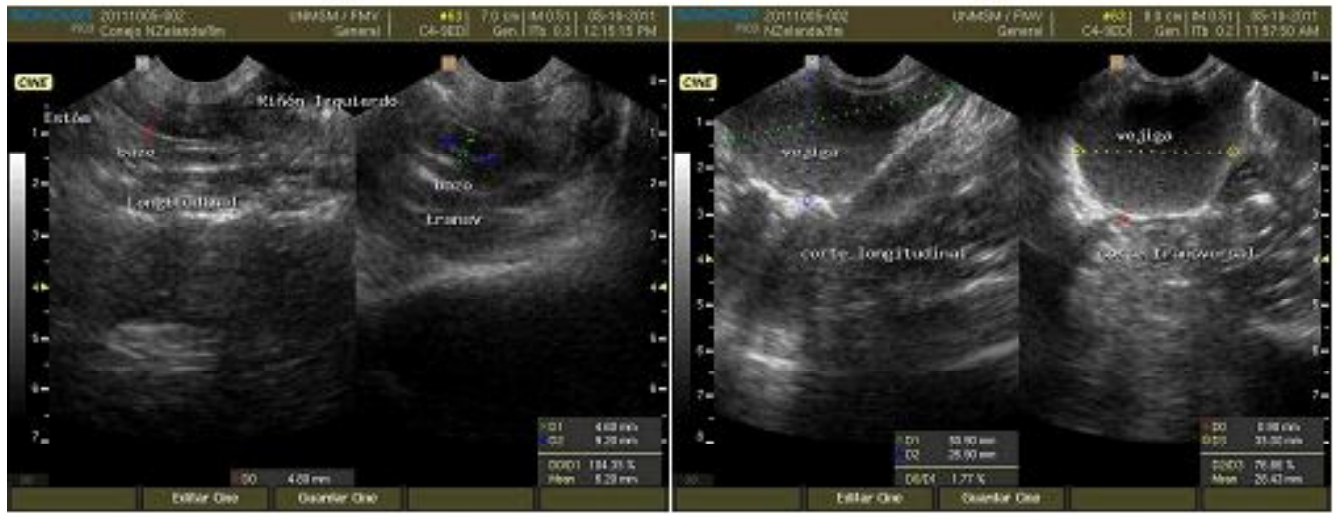

(D)

(C)

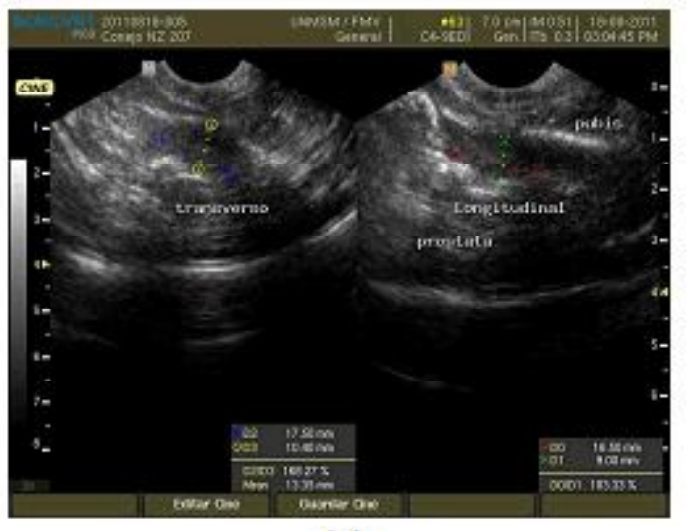

(E)

Figura 1. Imágenes ecográficas de órganos abdominales de conejos domésticos. A) Hígado en eje largo, mostrando los lóbulos derechos. En la imagen izquierda se observa la vesícula biliar y el estómago. En la imagen derecha se observa la vena cava y estómago, con el transductor ubicado hacia medial; B) Riñón derecho en plano sagital. Se observa la relación craneal con el hígado; C) Bazo en un corte longitudinal y transversal. En la imagen izquierda se observa su relación craneal con el estómago y caudal con el riñón izquierdo; D) Plano longitudinal y transverso de la vejiga urinaria normal. Observar la ecogenicidad incrementada de la orina por la presencia de sedimento en suspensión; E) Próstata en un corte transversal y longitudinal 
por lo que solo se evaluaba la pared ventral gástrica.

Los estudios sobre el patrón ecográfico normal de los conejos domésticos se enfocan principalmente en las características cualitativas de los órganos abdominales (Dimitrov et al., 2012b, 2013; Stamatova-Yovcheva et al., 2012), mientras que la información sobre las características cuantitativas es escasa. El presente estudio es un trabajo pionero en el país sobre el patrón ecográfico cuantitativo de conejos Nueva Zelanda, aunque dado lo limitado del tamaño muestral $(n=16)$, no se pude asumir que estos datos representan el patrón o rango normal.

Las medidas vesicales y gástricas halladas en este estudio están influenciadas por el moderado a abundante contenido de orina en vejiga y alimento en estómago, lo cual también podría ser un indicio de la buena salud de estos animales, si se considera que conejos enfermos usualmente presentan anorexia $\mathrm{y}$, en consecuencia, escaso contenido estomacal y vesical. Todos los conejos presentaron sedimento urinario vesical en grado variable.

Un resultado interesante fue encontrar que el grado de ecogenicidad en el hígado es mayor o semejante al de la corteza renal, y que el bazo es hipoecogénico con respecto al parénquima del hígado y la corteza renal.

El transductor microconvexo con frecuencia de 4 a $9 \mathrm{MHz}$ fue efectivo para visualizar los órganos abdominales de los animales examinados, el cual se recomienda para realizar el examen ecográfico abdominal de mamíferos pequeños (Redrobe, 2001; Reese, 2010).

Las medidas ecográficas renales obtenidas en el presente estudio difieren ligeramente de las medidas obtenidas por Dimitrov et al. (2012a) mediante examen post mortem, posiblemente debido a la metodología de medición empleada entre ambos estudios. Asimismo, las medidas renales fueron mayores que las reportadas por Reese (2010) en conejos de razas enanas, lo cual es un resultado esperado debido a la diferencia de tamaño entre los animales de los dos estudios.

\section{Literatura Citada}

1. Cardinalli R, Del Bosco A, Bonanno A, Di Grigoli A, Rebollar PG, Lorenzo PL, Castellini C. 2008. Connection between body condition score, chemical characteristics of body and reproductive traits of rabbit does. Livestock Sci 116: 209-215.

2. Dimitrov R, Kostov D, Stamatova K, Yordanova V. 2012a. Anatomotopographical and morphological analysis of normal kidneys of rabbit (Oryctolagus cuniculus). Trakia J Sci 10(2): 79-84.

3. Dimitrov R, Stamatova K, Russenov A, Kostov D, Vladova D, Stefanov M. 2012b. Ultrasonographic qualitative characters of rabbit spleen (Oryctolagus cuniculus). Trakia J Sci 10(1): 64-69.

4. Dimitrov R, Russenov A, StamatovaYovcheva K, Uzunova K, Yordanova $V$. 2013. Ultrasonographic characteristics of rabbit's pancreas. J Fac Vet Med Istanbul Univ 39(2):139-147.

5. Fischetti AJ. 2010. Diagnostic imaging. En: Quesenberry KE, Carpenter JW (eds). Ferrets, rabbits and rodents: clinical medicine and surgery. $3^{\text {rd }}$ ed. San Luis: Elsevier Saunders. p 502-510.

6. Graham J, Mader DR. 2012. Basic approach to veterinary care. En: Quesenberry KE, Carpenter JW (eds). Ferrets, rabbits and rodents: clinical medicine and surgery. $3^{\text {rd }}$ ed. San Luis: Elsevier Saunders. p 157-173.

7. Goddard PJ. 2000. Ecografía veterinaria. Zaragoza: Acribia. 387 p.

8. Harkness J, Wagner J. 1980. Biología y clínica de conejos y roedores. Zaragoza: Acribia. $182 \mathrm{p}$.

9. Lammers H, Winkelmann J. 1997. Enfermedades de los conejos. Zaragoza: Acribia. 154 p. 
10. Meredith A. 2000. Liver disease in rabbits. Semin Avian Exot Pet 19: 146-152.

11. Redrobe S. 2001. Imaging techniques in small mammals. Sem Avian Exot Pet 10: 187-197.

12. Redrobe S. 2006. Ultrasound of exotic species. En: Mannion P (ed). Diagnostic ultrasound in small animal practice. Oxford: Blackwell Science. p 301-329.

13. Reese S. 2010. Small mammals. In: Krautwald-Junghanns ME, Pees M, Reese S, Tully T (eds). Diagnostic imaging of exotic pets: birds, small mammals, reptiles. Hannover, Germany: Schlütersche Verlags. p 143-307.

14. Richardson VCG 2000. Husbandry. In: Richardson VCG (ed). Rabbits: health, husbandry and diseases. Oxford: Blackwell Science. p 1-6.

15. Stamatova-Yovcheva K, Dimitrov $R$, Yonkova P, Russenov A, Yovchev D, Kostov D. 2012. Comparative imaging anatomic study of domestic rabbit liver (Oryctolagus cuniculus). Trakia J Sci 10(1): 57-63.

16. Stein S, Walshaw S. 1996. Rabbits. In: Laber-Laird K, Swindle MM, Flecknell P (eds). Handbook of rodent and rabbit medicine. Oxford: Elsevier Science. $\mathrm{p}$ 183-218.

17. Van Der Hage M. 2000. Enfermedades del conejo. Tomo II. Madrid: MundiPrensa. p 549-587. 